\title{
Structure Elucidation and Antimicrobial Activities of Secondary Metabolites from the Flowery Parts of Verbascum mucronatum Lam.
}

\author{
Verbascum mucronatum Lam.'ın Çiçekli Kısımlarından Elde Edilen \\ Sekonder Metabolitlerin Yapı Tayini ve Antimikrobiyal Aktiviteleri
}

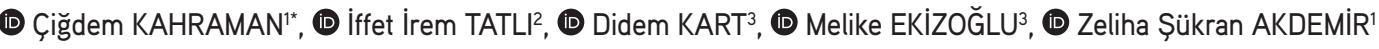 \\ 'Hacettepe University, Faculty of Pharmacy, Department of Pharmacognosy, Ankara, Turkey \\ ${ }^{2}$ Hacettepe University, Faculty of Pharmacy, Department of Pharmaceutical Botany, Ankara, Turkey \\ ${ }^{3}$ Hacettepe University, Faculty of Pharmacy, Department of Pharmaceutical Microbiology, Ankara, Turkey
}

\begin{abstract}
Objectives: To determine the secondary metabolites from Verbascum mucronatum Lam. and evaluate their antimicrobial activity.

Materials and Methods: Antimicrobial activities of the isolated metabolites were determined using broth microdilutions against the bacteria (Escherichia coli ATCC 25922, Enterococcus faecalis ATCC 29212, Pseudomonas aeruginosa ATCC 27853, Staphylococcus aureus ATCC 29213) and fungi (Candida albicans ATCC 90028, Candida krusei ATCC 6258, Candida parapsilosis ATCC 90018).

Results: Four iridoid glycosides; ajugol (1), aucubin (2), lasianthoside I (3), catalpol (4), two triterpenic saponins; ilwensisaponin C (5), ilwensisaponin A (=mimengoside A) (6), and one phenylethanoid glycoside; verbascoside (=acteoside) (7) were isolated from the water soluble parts of the methanolic extract gained flowery parts of $V$. mucronatum Lam.

Conclusion: Within the obtained compounds, ajugol and ilwensisaponin A showed moderate antimicrobial activity, especially against fungi.

Key words: Scrophulariaceae, Verbascum mucronatum Lam., secondary metabolites, antimicrobial activity
\end{abstract}

öz

Amaç: Bu çalışmada Verbascum mucronatum Lam.'ın sekonder metabolitlerinin belirlenmesi ve antimikrobiyal aktivitelerinin değerlendirilmesi amaçlanmıştır.

Gereç ve Yöntemler: İzole edilen metabolitlerin, bakteri (Escherichia coli ATCC 25922, Enterococcus faecalis ATCC 29212, Pseudomonas aeruginosa ATCC 27853, Staphylococcus aureus ATCC 29213) ve mantarlara (Candida albicans ATCC 90028, Candida krusei ATCC 6258, Candida parapsilosis ATCC 90018) karşı antimikrobiyal aktiviteleri sıvı mikrodilüsyon yöntemiyle belirlenmiștir.

Bulgular: V. mucronatum Lam.'ın çiçekli kısımlarının metanol ektresinin suda çözünen kısımlarından, dört iridoit glikoziti, ajugol (1), okubin (2), lasiantozit I (3), katalpol (4); iki triterpenik saponin, ilvensisaponin C (5), ilvensisaponin A (=mimengozit A) (6) ve bir feniletanoit glikoziti, verbaskozit (=akteozit) (7) izole edilmiștir.

Sonuç: Elde edilen bileșikler içinde ajugol ve ilvensisaponin A, özellikle mantarlara karşı zayıf antimikrobiyal aktivite göstermiştir.

Anahtar kelimeler: Scrophulariaceae, Verbascum mucronatum Lam., sekonder metabolitler, antimikrobiyal aktivite

\section{INTRODUCTION}

Verbascum is a widespread genus of the family Scrophulariaceae, which comprises more than 300 species of the world's flora. This genus is represented by 233 species, 196 of which are endemic in Turkish flora. ${ }^{2-4}$ Infusions prepared with the leaves and flowers of Verbascum species have been used as an expectorant and mucolytic ${ }^{5}$ wound healer ${ }^{6}$ for the treatment of hemorrhoids and rheumatism ${ }^{7}$ in folk medicine. Turker and Camper ${ }^{8}$ showed that Klebsiella pneumoniae and Staphylococcus aureus showed sensitivity to Mullein (Verbascum thapsus), which may explain why Mullein is used in folk medicine to

*Correspondence: E-mail: cigdemm@hacettepe.edu.tr, Phone: +90 5446227727 ORCID-ID: orcid.org/0000-0001-8096-0738 
treat respiratory disorders (caused by K. pneumoniae and $S$. aureus) and urinary tract infections (caused by K. pneumoniae). Antibacterial and antifungal activities of Verbascum L. species have been previously reviewed and the activity of the genus against several bacteria and fungi has been revealed. ${ }^{9}$ The antimicrobial activity of Verbascum mucronatum has also been determined using disc diffusion tests by our research group..$^{0}$ In addition, $V$. mucronatum Lam. has been used as a Hemostatic in Turkish traditional medicine."

Previous investigations on Turkish Verbascum L. species by our research group led to the isolation and characterization of a number of secondary metabolites such as iridoids, monoterpene glucosides, saponins, phenylethanoids, neolignans, and flavonoid glycosides. ${ }^{12-16}$ As a part of our ongoing studies on the secondary metabolites of Verbascum L. species, we have now investigated the methanolic extract of the flowery parts of $V$. mucronatum, and isolated four iridoids; ajugol (1), aucubin (2), lasianthoside I (3), catalpol (4), two saponins; ilwensisaponin C (5) and ilwensisaponin A (6), along with a phenylethanoid glycoside, verbascoside (=acteoside) (7) by means of various chromatographic techniques (Figure 1). The current paper deals with the isolation and structure elucidation of the compounds (1-7) from the title plant and the evaluation of their antimicrobial activities.

\section{MATERIALS AND METHODS}

\section{General experimental procedures}

The ultraviolet (UV) spectra $\left(\lambda_{\max }\right)$ were recorded on a Agilent 8453 spectrophotometer. The infrared (IR) spectra $\left(v_{\max }\right)$ were determined on a Perkin Elmer 2000 fourier transform (FT)-IR spectrophotometer. The 1D and 2D nuclear magnetic resonance (NMR) spectra were obtained on a Bruker Avance DRX 500 and $400 \mathrm{FT}$ spectrometer operating at 500 and $400 \mathrm{MHz}$ for ${ }^{1} \mathrm{H}$ NMR, and 125 and $100 \mathrm{MHz}$ for ${ }^{13} \mathrm{C}$ NMR. For the ${ }^{13} \mathrm{C}$ NMR spectra, multiplicities were determined using distortionless enhancement with a polarization transfer (DEPT) experiment. LC-ESIMS data were obtained using a Bruker BioApex FT-mass spectrometry instrument in the ESI mode. Reversed-phase material (C-18, LiChroprep 25-40 $\mu \mathrm{m}$ ) and polyamide were used for vacuum liquid chromatography (VLC), reversed-phase material (C-18, LiChroprep 25-40 $\mathrm{mm}$ ) was used for middle pressure liquid chromatography (MPLC), and Si gel (230-400 mesh) (Merck) was used for column chromatography (CC). Precoated silica gel $60 \mathrm{~F}_{254}$ aluminum sheets (Merck) were used for thin-layer chromatography (TLC); developing systems, $\mathrm{CHCl}_{3}$ $\mathrm{MeOH}-\mathrm{H}_{2} \mathrm{O}$ (61:32:7 and 80:20:2). Plates were examined using UV fluorescence and sprayed with $1 \%$ vanillin in concentrated $\mathrm{H}_{2} \mathrm{SO}_{4}$, followed by heating at $105^{\circ} \mathrm{C}$ for $1-2 \mathrm{~min}$.

\section{Plant material}

V. mucronatum Lam. was collected from Aksaray, $17 \mathrm{~km}$ from Aksaray to Ulukışla, in July 2007. A voucher specimen has been deposited in the Herbarium of the Faculty of Science, Gazi University, Ankara, Turkey (GAZI 10097). The flowery parts of the plant, which were air dried in the shade, were used in the phytochemical studies.

\section{Extraction and isolation}

Air-dried and powdered flowery parts of the plant $(586.2 \mathrm{~g}$ ) were extracted with $\mathrm{MeOH}(3 \times 2.5 \mathrm{~L})$. The $\mathrm{MeOH}$ extract was evaporated to dryness in vacuo to yield $70.4 \mathrm{~g}$ of crude extract, then $\mathrm{MeOH}$ extract was dissolved with $100 \mathrm{~mL}$ distilled water and partitioned in $\mathrm{CHCl}_{3}(2 \times 100 \mathrm{~mL}) . \mathrm{H}_{2} \mathrm{O}$ and $\mathrm{CHCl}_{3}$ phases were evaporated to dryness in vacuo to yield $65.8 \mathrm{~g} \mathrm{H}_{2} \mathrm{O}$ and $3.6 \mathrm{~g} \mathrm{CHCl}_{3}$ extracts. The $\mathrm{H}_{2} \mathrm{O}$ phase was fractionated using $\mathrm{CC}$ on polyamide $\left(150 \mathrm{~g}\right.$ ) using $\mathrm{H}_{2} \mathrm{O}-\mathrm{MeOH}(100: 0 \rightarrow 0: 100)$ (each $500 \mathrm{~mL}$ ), respectively, to yield 6 fractions (Frs. A-F). Fraction D $(4.9 \mathrm{~g})$, eluted with $75 \%$ methanol, was subjected to VLC using reversed-phase material (C-18, LiChroprep 25-40 $\mu \mathrm{m}, 150 \mathrm{~g}$ ), using $\mathrm{MeOH}-\mathrm{H}_{2} \mathrm{O}$ mixtures (0-100\%) to give catalpol (4) (62.1 $\mathrm{mg}$ ), aucubin (2) (139.3 mg), ajugol (1) (48.6 mg), Fr. D3 (1.19 g) and Fr. D4 (625.3 mg). Frs. D3 and D4 were rechromatographed. Fr. D3 was applied to MPLC using reversed-phase material (C-18, LiChroprep 25-40 $\mu \mathrm{m}$ ) using $\mathrm{MeOH}-\mathrm{H}_{2} \mathrm{O}$ mixtures (100:0 $\rightarrow 30$ 70) to yield ilwensisaponin C (5) (14.7 mg), ilwensisaponin A (6) (51.5 mg), and lasianthoside I (3) (6.7 mg). Fr. D4 was rechromatographed on a silica gel column $(55 \mathrm{mg})$ and eluted $\mathrm{CHCl}_{3}-\mathrm{MeOH}(70: 30 \rightarrow 60: 40)$ mixtures to give verbascoside (=acteoside) (7) (14.8 mg).

\section{Antimicrobial activity-broth microdilution method}

Antibacterial and antifungal activities were determined using the broth microdilution test as recommended by Clinical and Laboratory Standards Institute. 17,18 Plant extracts were tested against four bacteria including two Gram-positive (S. aureus ATCC 29213, Enterococcus faecalis ATCC 29212) and two Gram-negative microorganisms (Escherichia coli ATCC 25922, Pseudomonas aeruginosa ATCC 27853), as well as for antifungal activities against three yeasts (Candida albicans ATCC 90028, Candida krusei ATCC 6258, Candida parapsilosis ATCC 90018). The antibacterial activity test was performed in Mueller-Hinton broth (MHB, Difco Laboratories, Detroit, MI, USA); for antifungal test, RPMI-1640 medium with L-glutamine (ICN-Flow, Aurora, OH, USA), buffered with MOPS buffer (ICN-Flow, Aurora, OH, USA) was used. The inoculum densities were approximately $5 \times 10^{5} \mathrm{CFU} / \mathrm{mL}$ and 0.5 $2.5 \times 10^{3} \mathrm{CFU} / \mathrm{mL}$ for bacteria and fungi, respectively.

Each plant extract was dissolved in $2.44 \mathrm{~mL}$ DMSO. Finally, two-fold concentrations were prepared in the wells of the microtiter plates, between 1024-1 $\mu \mathrm{g} / \mathrm{mL}$. Ampicillin and fluconazole were used as reference antibiotics for bacteria and fungi, respectively $(64-0.0625 \mu \mathrm{g} / \mathrm{mL})$. Microtiter plates were incubated at $35^{\circ} \mathrm{C}$ for $18-24 \mathrm{~h}$ for bacteria and $48 \mathrm{~h}$ for fungi. After the incubation period, minimum inhibitory concentration (MIC) values were defined as the lowest concentration of the extracts that inhibits the visible growth of the microorganisms.

\section{RESULTS}

Ajugol (1): UV $\lambda_{\text {max }}(\mathrm{MeOH}) 220 \mathrm{~nm}, \mathrm{IR}(\mathrm{KBr}) v_{\max } 3410(\mathrm{OH})$, $1660(\mathrm{C}=\mathrm{C}) \mathrm{cm}^{-1}$, Positive ion LC-ESIMS m/z $371[\mathrm{M}+\mathrm{Na}]^{+}$(calc. for $\left.\mathrm{C}_{15} \mathrm{H}_{24} \mathrm{O}_{9}\right),{ }^{1} \mathrm{H}$ NMR (400 MHz, DMSO- $\left.d_{6}\right)$ of 1: $\delta_{\mathrm{H}} 6.10(1 \mathrm{H}$, dd, J=6/1.6 Hz, H-3), $5.29(1 \mathrm{H}, \mathrm{d}, \mathrm{J}=2 \mathrm{~Hz}, \mathrm{H}-1), 4.78(1 \mathrm{H}, \mathrm{dd}$, $J=6 / 2.8 \mathrm{~Hz}, \mathrm{H}-4), 4.43\left(1 \mathrm{H}, \mathrm{d}, J=7.6 \mathrm{~Hz}, \mathrm{H}-1^{\prime}\right), 3.71(1 \mathrm{H}, \mathrm{d}, J=2.8$ $\mathrm{Hz}, \mathrm{H}-6), 3.71-3.65$ (2H, *, H-6'), 3.05-2.93 (1H, *, H-2', H-3', $\left.\mathrm{H}-4^{\prime}, \mathrm{H}-5^{\prime}\right), 2.47(1 \mathrm{H}, \mathrm{m}, \mathrm{H}-5), 2.32(1 \mathrm{H}, \mathrm{t}, \mathrm{J}=10 \mathrm{~Hz}, \mathrm{H}-9), 1.84(1 \mathrm{H}$, 

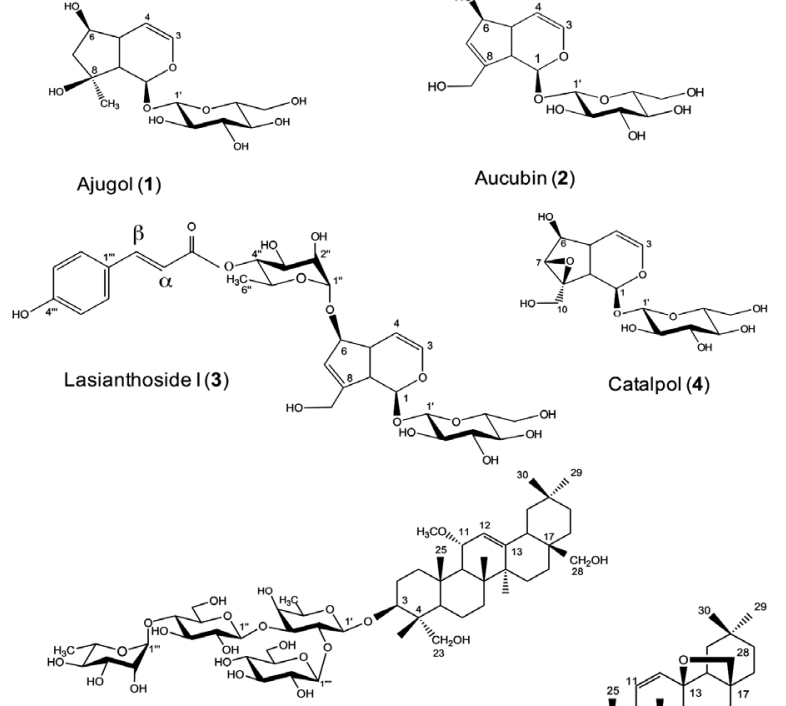

Ilwensisaponin C (5)
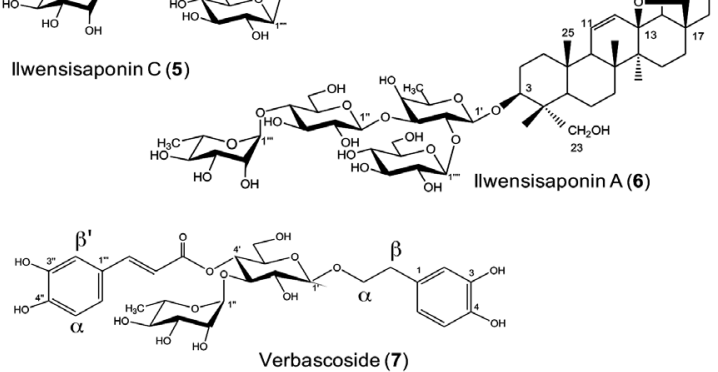

Figure 1. Isolated secondary metabolites from Verbascum mucronatum Lam.

Table 1. ${ }^{13} \mathrm{C}$ NMR (DMSO- $\mathrm{d}_{6}$ ) data of compounds of $1,2,3$ and 4

\begin{tabular}{lllll}
\hline & $2(100 \mathrm{MHz})$ & $3(125 \mathrm{MHz})$ & $4(100 \mathrm{MHz})$ & $1(100 \mathrm{MHz})$ \\
\hline $\mathrm{C} / \mathrm{H}$ atom & $\delta_{\mathrm{C}}(\mathrm{ppm})$ & $\delta_{\mathrm{C}}(\mathrm{ppm})$ & $\delta_{\mathrm{C}}(\mathrm{ppm})$ & $\delta_{\mathrm{C}}(\mathrm{ppm})$ \\
\hline Aglycone & & & & \\
\hline 1 & 95.9 & 96.0 & 93.8 & 92.1 \\
\hline 3 & 140.6 & 141.1 & 140.7 & 139.3 \\
\hline 4 & 105.6 & 104.8 & 103.8 & 105.7 \\
\hline 5 & 45.2 & 42.8 & 37.8 & 40.7 \\
\hline 6 & 81.1 & 87.5 & 77.8 & 77.8 \\
\hline 7 & 129.8 & 125.6 & 61.2 & 50.6 \\
\hline 8 & 146.8 & 149.4 & 65.3 & 77.7 \\
\hline 9 & 47.0 & 47.3 & 42.6 & 50.5 \\
\hline 10 & 60.1 & 59.9 & 59.5 & 25.7 \\
\hline
\end{tabular}

Glc at $\mathrm{C}-1$

\begin{tabular}{lllll}
\hline $1^{\prime}$ & 98.7 & 100.1 & 98.3 & 98.1 \\
\hline $2^{\prime}$ & 74.0 & 73.9 & 73.8 & 73.8 \\
\hline $3^{\prime}$ & 77.3 & 77.2 & 76.8 & 76.1 \\
\hline $4^{\prime}$ & 70.7 & 70.7 & 70.6 & 70.7 \\
\hline $5^{\prime}$ & 77.8 & 77.6 & 77.6 & 77.4 \\
\hline $6^{\prime}$ & 61.7 & 61.6 & 61.7 & 61.7 \\
\hline
\end{tabular}

Compound 3: Rha at C-6, 98.6 (C-1"), 74.3 (C4"), 71.4 (C-2"), 68.8 (C-3"), 67.0 (5"), 18.0 (C-6"); Acyl moiety, 166.8 (C=0), 161.0 (C-4"'), 145.2 (C- $\beta$ ), 133.0 (1'"), 130.7 (C-2"'), 130.7 (C-6"'), 116.3 (C-3"'), 116.3 (C-5"'), 115.4 (C- $\alpha$ ) dd, J=12.8/6.0 Hz, H-7b), $1.63(1 \mathrm{H}, \mathrm{dd}, J=13.2 / 6.0 \mathrm{~Hz}, \mathrm{H}-7 \mathrm{a}$ ), 1.13 $(3 \mathrm{H}, \mathrm{s}, \mathrm{H}-10)$, and ${ }^{13} \mathrm{C}$ NMR $\left(100 \mathrm{MHz}\right.$, DMSO- $d_{6}$ ) (see Table 1).

Aucubin (2): UV $\lambda_{\max }(\mathrm{MeOH}) 205 \mathrm{~nm},(\mathrm{KBr}) v_{\max } 3275(\mathrm{OH})$, $1650(\mathrm{C}=\mathrm{C}) \mathrm{cm}^{-1}$, Positive ion LC-ESIMS m/z $369[\mathrm{M}+\mathrm{Na}]^{+}$(calc. for $\left.\mathrm{C}_{15} \mathrm{H}_{22} \mathrm{O}_{9}\right),{ }^{1} \mathrm{H}$ NMR $\left(400 \mathrm{MHz}\right.$, DMSO- $\left.d_{6}\right)$ of $2: \delta_{\mathrm{H}} 6.30(1 \mathrm{H}, \mathrm{dd}$, $J=4.8 / 1.6 \mathrm{~Hz}, \mathrm{H}-3), 5.65(1 \mathrm{H}, \mathrm{bs}, \mathrm{H}-7) 5.01(1 \mathrm{H}, \mathrm{d}, \mathrm{J}=4.8 \mathrm{~Hz}, \mathrm{H}-4)$, $4.95(1 \mathrm{H}, \mathrm{d}, J=5.6 \mathrm{~Hz}, \mathrm{H}-1), 4.85\left(1 \mathrm{H}, \mathrm{d}, J=7.7 \mathrm{~Hz}, \mathrm{H}-1^{\prime}\right), 4.40,(1 \mathrm{H}$, d, J=6.4 Hz, H-6), $4.14(1 \mathrm{H}, \mathrm{dd}, J=12.4 / 4.0 \mathrm{~Hz}, \mathrm{H}-10 \mathrm{~b}), 3.96(1 \mathrm{H}$, dd, J=12.4/4.0 Hz, H-10a), 3.66 (1H, dd, J=12.8/4.8 Hz, H-6'a), $3.42\left(1 \mathrm{H}, \mathrm{dd}, \mathrm{J}=12.0 / 4.8 \mathrm{~Hz}, \mathrm{H}-6^{\prime} \mathrm{b}\right), 3.16\left(1 \mathrm{H}, \mathrm{m}, \mathrm{H}-3^{\prime}\right), 3.11(1 \mathrm{H}$, m, H-4'), $3.04\left(1 \mathrm{H}, \mathrm{m}, \mathrm{H}-5^{\prime}\right), 3.00\left(1 \mathrm{H}, \mathrm{m}, \mathrm{H}-2^{\prime}\right), 2.72(1 \mathrm{H}, \mathrm{t}, \mathrm{J}=7.2$ $\mathrm{Hz}, \mathrm{H}-9), 2.50(1 \mathrm{H}, \mathrm{m}, \mathrm{H}-5)$, and ${ }^{13} \mathrm{C}$ NMR $\left(100 \mathrm{MHz}, \mathrm{DMSO}-d_{6}\right)$ (see Table 1).

Lasianthoside I (3): UV $\lambda_{\text {max }}(\mathrm{MeOH}) 216,277 \mathrm{~nm}, \mathrm{IR}(\mathrm{KBr}) v_{\text {max }}$ $3405(\mathrm{OH}), 1704(\mathrm{C}=0), 1655(\mathrm{C}=\mathrm{C}), 1508,1451$ (aromatic ring) $\mathrm{cm}^{-1}$, Positive ion LC-ESIMS m/z $611[\mathrm{M}+\mathrm{Na}]^{+}$(calc. for $\mathrm{C}_{30} \mathrm{H}_{38} \mathrm{O}_{15}$ ), ${ }^{1} \mathrm{H}$ NMR $\left(400 \mathrm{MHz}\right.$, DMSO- $\left.d_{6}\right)$ of $3: \delta_{H} 6.37(1 \mathrm{H}, \mathrm{dd}, J=4.8 / 1.2 \mathrm{~Hz}$, $\mathrm{H}-3), 5.26(1 \mathrm{H}, \mathrm{d}, J=4.4 \mathrm{~Hz}, \mathrm{H}-4), 5.10(1 \mathrm{H}, \mathrm{d}, J=4.0 \mathrm{~Hz}, \mathrm{H}-1), 4.91$ $\left(1 \mathrm{H}, \mathrm{d}, J=7.6 \mathrm{~Hz}, \mathrm{H}-1^{\prime}\right), 4.18(1 \mathrm{H}, \mathrm{d}, \mathrm{J}=6.0 \mathrm{~Hz}, \mathrm{H}-10 \mathrm{~b}), 3.86(1 \mathrm{H}, \mathrm{d}$, $J=4 \mathrm{~Hz}, \mathrm{H}-6$ 'b), $3.78(1 \mathrm{H}, \mathrm{t}, \mathrm{J}=6.8 \mathrm{~Hz}, \mathrm{H}-6), 3.66\left(1 \mathrm{H},{ }^{*}, \mathrm{H}-10 \mathrm{a}\right)$, $3.64\left(1 \mathrm{H}, \mathrm{dd}, J=10.8 / 6.4 \mathrm{~Hz}, \mathrm{H}-6{ }^{\prime} \mathrm{a}\right), 3.35(1 \mathrm{H}, \mathrm{s}, \mathrm{H}-7), 2.31(1 \mathrm{H}$, t, J=7.6 Hz, H-9), 3.13-3.19 (1H, *, H-3', H-4', H-5'), $3.02(1 \mathrm{H}, \mathrm{dd}$, $\left.J=10 / 6.4 \mathrm{~Hz}, \mathrm{H}-2^{\prime}\right), 2.12(1 \mathrm{H}, \mathrm{m}, \mathrm{H}-5)$, and ${ }^{13} \mathrm{C}$ NMR $(125 \mathrm{MHz}$, DMSO- $d_{6}$ ) (see Table 1).

Catalpol (4): UV $\lambda_{\text {max }}(\mathrm{MeOH}) \mathrm{nm} 208 \mathrm{~nm}, \mathrm{IR}(\mathrm{KBr}) v_{\max } 3450$ $(\mathrm{OH}), 1670(\mathrm{C}=\mathrm{C}) \mathrm{cm}^{-1}$, Positive ion LC-ESIMS m/z $385[\mathrm{M}+\mathrm{Na}]^{+}$ (calc. for $\mathrm{C}_{15} \mathrm{H}_{22} \mathrm{O}_{10}$ ), ${ }^{1} \mathrm{H}$ NMR $\left(400 \mathrm{MHz}\right.$, DMSO- $\left.d_{6}\right)$ of $4: \delta_{\mathrm{H}} 6.37$ $(1 \mathrm{H}, \mathrm{dd}, J=4.8 / 1.2 \mathrm{~Hz}, \mathrm{H}-3), 5.26(1 \mathrm{H}, \mathrm{d}, J=4.4 \mathrm{~Hz}, \mathrm{H}-4), 5.10(1 \mathrm{H}$, d, J=4.0 Hz, H-1), 4.91, (1H, d, J=7.6 Hz, H-1'), $4.18(1 \mathrm{H}, d, J=6.0$ $\mathrm{Hz}, \mathrm{H}-10 \mathrm{~b}), 3.86\left(1 \mathrm{H}, \mathrm{d}, J=4 \mathrm{~Hz} H-6^{\prime} b\right), 3.78(1 \mathrm{H}, \mathrm{t}, J=6.8 \mathrm{~Hz}$, H-6), $3.66(1 \mathrm{H}, *, 10 \mathrm{a}), 3.64\left(1 \mathrm{H}, \mathrm{dd}, J=10.8 / 6.4 \mathrm{~Hz}, \mathrm{H}-6{ }^{\prime} \mathrm{a}\right), 3.35$ (1H, s, H-7), 3.13-3.19 ( $\left.{ }^{*}, \mathrm{H}-3^{\prime}, \mathrm{H}-4^{\prime}, \mathrm{H}-5^{\prime}\right), 3.02(1 \mathrm{H}, \mathrm{dd}, \mathrm{J}=10 / 6.4$ $\left.\mathrm{Hz}, \mathrm{H}-2^{\prime}\right), 2.31(1 \mathrm{H}, \mathrm{t}, \mathrm{J}=7.6 \mathrm{~Hz}, \mathrm{H}-9), 2.12(1 \mathrm{H}, \mathrm{m}, \mathrm{H}-5)$, and ${ }^{13} \mathrm{C}$ NMR (100 MHz, DMSO- $d_{6}$ ) (see Table 1).

Ilwensisaponin C (5): UV $\lambda_{\text {max }}(\mathrm{MeOH}) 205 \mathrm{~nm}, \mathrm{IR}(\mathrm{KBr}) v_{\max }$ $3400(\mathrm{OH}), 1665(\mathrm{C}=\mathrm{C}) \mathrm{cm}^{-1}$, Positive ion LC-ESIMS m/z 1127 [M+Na] $]^{+}$(calc. for $\mathrm{C}_{55} \mathrm{H}_{92} \mathrm{O}_{22}$ ), ${ }^{1} \mathrm{H}$ NMR (400 MHz, pyridine) of 5 : $\delta_{\mathrm{H}} 5.78\left(1 \mathrm{H}, \mathrm{bs}, \mathrm{H}-1^{\prime \prime \prime}\right), 5.54\left(1 \mathrm{H}, \mathrm{d}, \mathrm{J}=7.0 \mathrm{~Hz}, \mathrm{H}-1^{\prime \prime \prime \prime}\right), 5.46(1 \mathrm{H}$, bs, $H-12), 5.21\left(1 \mathrm{H}, d, J=7.0 \mathrm{~Hz}, \mathrm{H}-1^{\prime \prime}\right), 4.91(1 \mathrm{H}, \mathrm{d}, J=6.6 \mathrm{~Hz}$, $\left.\mathrm{H}-1^{\prime}\right), 4.35\left(1 \mathrm{H},{ }^{*}, \mathrm{H}-2^{\prime}\right), 4.33\left(1 \mathrm{H},{ }^{*}, \mathrm{H}-23 \mathrm{~b}\right), 4.10\left(1 \mathrm{H},{ }^{*}, \mathrm{H}-\mathrm{2}^{\prime \prime \prime \prime}\right)$, $4.10\left(1 \mathrm{H},{ }^{*}, \mathrm{H}-3\right), 3.89\left(1 \mathrm{H},{ }^{*}, \mathrm{H}-2{ }^{\prime \prime}\right), 3.82\left(1 \mathrm{H},{ }^{*}, \mathrm{H}-11\right), 3.81(1 \mathrm{H}$, d, J=11.7 Hz, H-28b), $3.69(1 \mathrm{H}, d, J=8.3 \mathrm{~Hz}, \mathrm{H}-23 \mathrm{a}), 3.57(1 \mathrm{H}, \mathrm{d}$, $J=10.2 \mathrm{~Hz}, \mathrm{H}-28 \mathrm{a}), 1.68\left(3 \mathrm{H}, \mathrm{d}, J=5.5 \mathrm{~Hz}, \mathrm{H}-6^{\prime \prime \prime}\right), 1.35$ (3H, d, $\left.J=4.8 \mathrm{~Hz}, \mathrm{H}-6^{\prime}\right), 1.30(3 \mathrm{H}, \mathrm{s}, \mathrm{H}-27), 1.08(3 \mathrm{H}, \mathrm{s}, \mathrm{H}-24), 1.07(3 \mathrm{H}$, $\mathrm{s}, \mathrm{H}-25), 0.96(3 \mathrm{H}, \mathrm{s}, \mathrm{H}-26), 0.95(3 \mathrm{H}, \mathrm{s}, \mathrm{H}-30), 0.88(3 \mathrm{H}, \mathrm{s}$, $\mathrm{H}-29), \mathrm{CH}_{3} \mathrm{O}: 3.21(3 \mathrm{H}, \mathrm{s})$, and ${ }^{13} \mathrm{C}$ NMR $(125 \mathrm{MHz}$, pyridine) (see Table 2).

Ilwensisaponin A (6): UV $\lambda_{\text {max }}(\mathrm{MeOH}) 206 \mathrm{~nm}, \mathrm{IR}(\mathrm{KBr}) v_{\text {max }}$ $3434(\mathrm{OH}), 1645(\mathrm{C}=\mathrm{C}) \mathrm{cm}^{-1}$, Positive ion LC-ESIMS m/z 1095 $[\mathrm{M}+\mathrm{Na}]^{+}$(calc. for $\left.\mathrm{C}_{54} \mathrm{H}_{88} \mathrm{O}_{21}\right),{ }^{1} \mathrm{H}$ NMR $(500 \mathrm{MHz}$, pyridine) of 6: $\delta_{H} 5.94(1 \mathrm{H}, \mathrm{d}, J=10.4 \mathrm{~Hz}, \mathrm{H}-11), 5.77\left(1 \mathrm{H}, \mathrm{d}, J=1.5 \mathrm{~Hz}, \mathrm{H}-1^{\prime \prime \prime}\right), 5.53$ $\left(1 \mathrm{H},{ }^{*}, \mathrm{H}-12\right), 5.20\left(1 \mathrm{H}, \mathrm{d}, J=7.6 \mathrm{~Hz}, \mathrm{H}-1^{\prime \prime}\right), 5.53(1 \mathrm{H}, \mathrm{d}, J=7.9 \mathrm{~Hz}$, $\mathrm{H}-1^{\prime \prime \prime \prime \prime), ~} 4.91\left(1 \mathrm{H}, \mathrm{d}, \mathrm{J}=7.7 \mathrm{~Hz}, \mathrm{H}-1^{\prime}\right), 4.58\left(1 \mathrm{H},{ }^{*}, \mathrm{H}-2^{\prime \prime \prime}\right), 4.34(1 \mathrm{H}$, *, H-23b), $4.25\left(1 \mathrm{H},{ }^{*}, \mathrm{H}-\mathrm{2}^{\prime}\right), 4.11\left(1 \mathrm{H},{ }^{*}, \mathrm{H}-3\right), 4.05\left(1 \mathrm{H},{ }^{*}, \mathrm{H}-\mathrm{2}^{\prime \prime \prime \prime}\right)$, $3.90\left(1 \mathrm{H},{ }^{*}, \mathrm{H}-2{ }^{\prime \prime}\right), 3.72\left(1 \mathrm{H},{ }^{*}, \mathrm{H}-28 \mathrm{~b}\right), 3.70\left(1 \mathrm{H},{ }^{*}, \mathrm{H}-23 \mathrm{a}\right), 3.33$ 
Table $2 .{ }^{13} \mathrm{C}$ NMR (125 MHz, pyridine-d5/5, CD $\mathrm{OD} / 6$ ) data of compounds 5 and 6

\begin{tabular}{|c|c|c|c|c|c|}
\hline & 5 & 6 & & 5 & 6 \\
\hline $\mathrm{C} / \mathrm{H}$ atom & $\delta_{C}(\mathbf{p p m})$ & $\delta_{C}(\mathbf{p p m})$ & C/atom & $\delta_{C}(p p m)$ & $\delta_{C}(\mathrm{ppm})$ \\
\hline Aglycone & & & Sugar units & & \\
\hline 1 & 40.2 & 38.0 & Fuc at C-3 & & \\
\hline 2 & 22.9 & 25.6 & $1^{\prime}$ & 104.2 & 104.7 \\
\hline 3 & 83.0 & 84.0 & $2^{\prime}$ & 77.0 & 77.1 \\
\hline 4 & 44.1 & 45.9 & $3^{\prime}$ & 85.0 & 85.7 \\
\hline 5 & 48.1 & 46.0 & $4^{\prime}$ & 72.2 & 72.2 \\
\hline 6 & 18.5 & 18.0 & $5^{\prime}$ & 70.6 & 70.7 \\
\hline 7 & 31.9 & 31.0 & $6^{\prime}$ & 17.3 & 17.0 \\
\hline 8 & 37.6 & 42.6 & Glc at Fuc C-3' & & \\
\hline 9 & 52.8 & 54.1 & $1^{\prime \prime}$ & 105.1 & 105.1 \\
\hline 10 & 35.8 & 37.0 & 2" & 75.6 & 75.4 \\
\hline 11 & 76.2 & 132.9 & $3 "$ & 77.8 & 76.1 \\
\hline 12 & 122.6 & 131.9 & 4" & 78.4 & 79.3 \\
\hline 13 & 148.1 & 86.9 & $5 "$ & 77.2 & 76.4 \\
\hline 14 & 43.6 & 44.1 & $6 "$ & 61.4 & 63.5 \\
\hline 15 & 26.7 & 26.0 & Rha at Glc C-4" & & \\
\hline 16 & 26.4 & 26.5 & 1"' & 102.8 & 102.9 \\
\hline 17 & 42.2 & 40.0 & $2 " '$ & 72.8 & 72.7 \\
\hline 18 & 42.5 & 52.8 & 3"' & 72.6 & 71.3 \\
\hline 19 & 47.1 & 38.3 & 4"' & 74.0 & 73.8 \\
\hline 20 & 31.4 & 31.0 & 5"' & 70.5 & 70.7 \\
\hline 21 & 33.3 & 34.0 & 6"' & 18.5 & 18.5 \\
\hline 22 & 34.8 & 32.0 & Glc at Fuc C-2' & & \\
\hline 23 & 64.8 & 64.5 & $1 " \prime \prime$ & 104.0 & 103.5 \\
\hline 24 & 13.4 & 12.6 & 2"'” & 76.2 & 75.4 \\
\hline 25 & 18.0 & 19.0 & 3"'” & 78.8 & 76.8 \\
\hline 26 & 18.7 & 22.0 & 4"'” & 72.2 & 73.5 \\
\hline 27 & 26.4 & 20.0 & $5 " \prime$ & 76.5 & 78.3 \\
\hline 28 & 68.9 & 78.3 & 6"'” & 63.3 & 61.8 \\
\hline 29 & 33.5 & 34.0 & & & \\
\hline 30 & 24.0 & 24.0 & & & \\
\hline $\mathrm{OCH}_{3}$ & 54.1 & - & & & \\
\hline
\end{tabular}

(1H, d, J=6.2 Hz, H-28a), $1.68\left(1 \mathrm{H}, \mathrm{d}, J=6.1 \mathrm{~Hz}, \mathrm{H}-6^{\prime \prime \prime}\right), 1.38(3 \mathrm{H}$, bs, H-6'), $1.31(3 \mathrm{H}, \mathrm{s}, \mathrm{H}-26), 1.04(3 \mathrm{H}, \mathrm{s}, \mathrm{H}-24), 0.98(3 \mathrm{H}, \mathrm{s}, \mathrm{H}-27)$, $0.96(3 \mathrm{H}, \mathrm{s}, \mathrm{H}-25), 0.87(3 \mathrm{H}, \mathrm{s}, \mathrm{H}-29), 0.82(3 \mathrm{H}, \mathrm{s}, \mathrm{H}-30)$, and ${ }^{13} \mathrm{C}$ NMR (125 MHz, CD 3 OD) (see Table 2).

Verbascoside (Acteoside) (7): UV $\lambda_{\max }(\mathrm{MeOH}) 220,332 \mathrm{~nm}$, IR $(\mathrm{KBr}) v_{\max } 3392(\mathrm{OH}), 1699(\mathrm{C}=\mathrm{O}), 1631(\mathrm{C}=\mathrm{C}), 1604,1525$ (aromatic ring) $\mathrm{cm}^{-1}$, Positive ion LC-ESIMS m/z $647[\mathrm{M}+\mathrm{Na}]^{+}$ (calc. for $\mathrm{C}_{29} \mathrm{H}_{36} \mathrm{O}_{15}$ ), ${ }^{1} \mathrm{H}$ NMR (500 MHz, DMSO- $d_{6}$ ) of $7: \delta_{H} 7.48$ $\left(1 \mathrm{H}, \mathrm{d}, J=15.8 \mathrm{~Hz}, \mathrm{H}-\beta^{\prime}\right), 7.04\left(1 \mathrm{H}, \mathrm{s}, \mathrm{H}-2^{\prime \prime \prime}\right), 6.97(1 \mathrm{H}, \mathrm{d}, J=7.5$
Hz, H-6"') 6.79 (1H, d, J=7.7 Hz, H-5"'), 6.67 (1H, bs, H-2), 6.67 $(1 \mathrm{H}$, bs, H-5), $6.52(1 \mathrm{H}, \mathrm{d}, J=7.5 \mathrm{~Hz}, \mathrm{H}-6), 6.20(1 \mathrm{H}, \mathrm{d}, J=15.8 \mathrm{~Hz}$, $\left.H-\alpha^{\prime}\right), 5.07\left(1 \mathrm{H}, \mathrm{bs}, \mathrm{H}-1^{\prime \prime}\right), 4.75\left(1 \mathrm{H}, \mathrm{t}, \mathrm{J}=9.4 \mathrm{~Hz}, \mathrm{H}-4^{\prime}\right), 4.37(1 \mathrm{H}$, d, J=7.7 Hz, H-1'), $3.72\left(1 \mathrm{H},{ }^{*}, \mathrm{H}-2^{\prime \prime}\right), 3.91,\left(1 \mathrm{H}, \mathrm{m}, \mathrm{H}-\alpha_{\mathrm{b}}\right), 3.67,(1 \mathrm{H}$, $\left.\mathrm{m}, \mathrm{H}-\alpha_{\mathrm{a}}\right), 2.73(2 \mathrm{H}, \mathrm{s}, \mathrm{H}-\beta), 3.68\left(1 \mathrm{H},{ }^{*}, \mathrm{H}-3^{\prime}\right), 3.45-3.70\left(2 \mathrm{H},{ }^{*}\right.$, $\mathrm{H}-6$ '), $3.45\left(1 \mathrm{H},{ }^{*}, \mathrm{H}-5^{\prime}\right), 3.36\left(1 \mathrm{H},{ }^{*}, \mathrm{H}-5^{\prime \prime}\right), 3.35\left(1 \mathrm{H},{ }^{*}, \mathrm{H}-3^{\prime \prime}\right)$, $3.26\left(1 \mathrm{H}, \mathrm{t}, \mathrm{J}=8.3 \mathrm{~Hz}, \mathrm{H}-2^{\prime}\right), 3.15\left(1 \mathrm{H},{ }^{*}, \mathrm{H}-4^{\prime \prime}\right), 1.00(3 \mathrm{H}, \mathrm{d}, \mathrm{J}=5.8$ $\mathrm{Hz}, \mathrm{H}-6 "$ ), and ${ }^{13} \mathrm{C}$ NMR (125 $\mathrm{MHz} \mathrm{CDCl}_{3}$ ) (see Table 3).

*(overlapped) 
The methanolic extract of the flowery part of $V$. mucronatum and isolated compounds possessed moderate antimicrobial activity, especially against fungi. Iridoid glycoside ajugol was found to be the most active compound against $C$. albicans and $C$. parapsilosis with an MIC value of $64 \mu \mathrm{g} / \mathrm{mL}$, as well as ilwensisaponin A

Table $3 .{ }^{13} \mathrm{C}$ NMR $\left(125 \mathrm{MHz}, \mathrm{CDCl}_{3}\right.$ ) data of compound 7

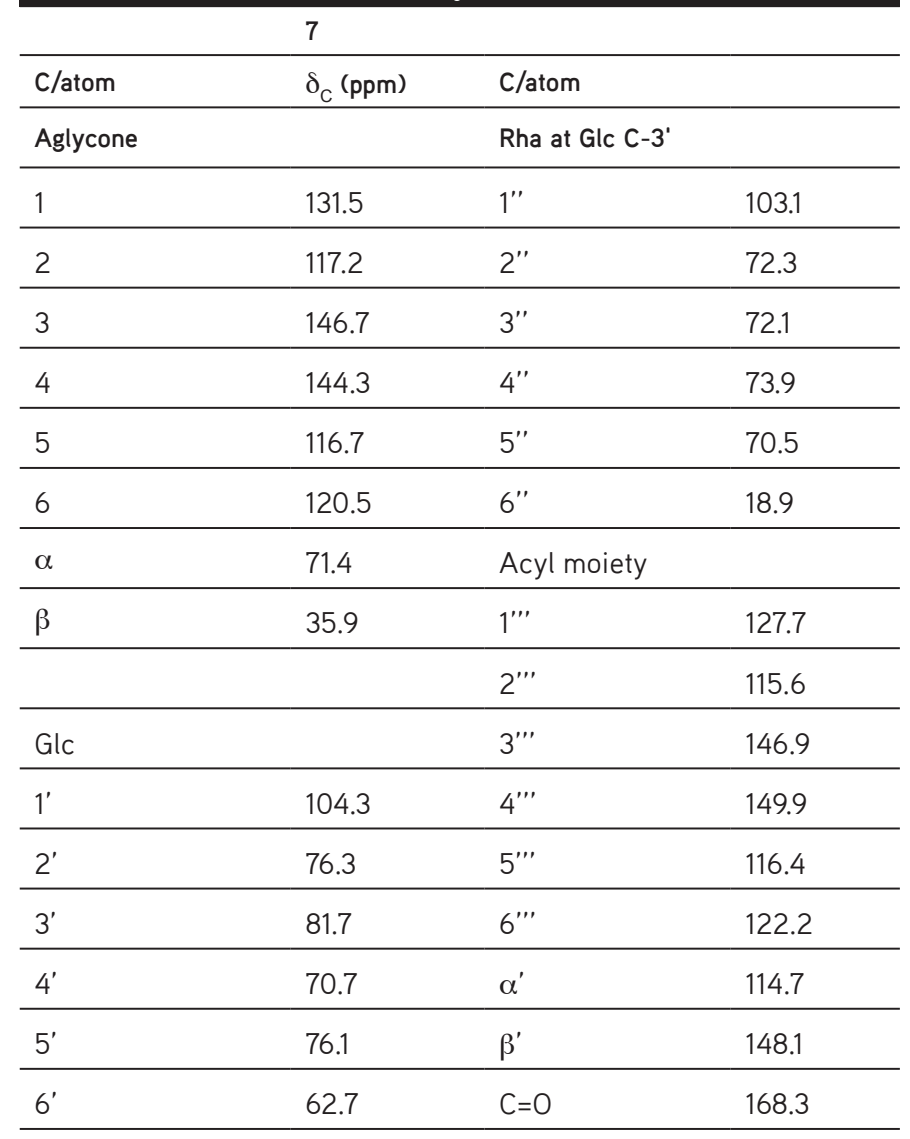

inhibited C. albicans and C. krusei with the same MIC value as ajugol. These active compounds were found to be much more effective against fungi than the $V$. mucronatum extract (Table 4).

\section{DISCUSSION}

Compound 1 was isolated as a white amorphous powder with the molecular formula $\mathrm{C}_{15} \mathrm{H}_{24} \mathrm{O}_{9}\left(\mathrm{LC}-\right.$ ESIMS $\left.\mathrm{m} / \mathrm{z} 371[\mathrm{M}+\mathrm{Na}]^{+}\right)$. An iridoid enolether system (220 nm) in UV spectrum; hydroxyl group (3410 $\left.\mathrm{cm}^{-1}\right)$ and double-bond $\left(1660 \mathrm{~cm}^{-1}\right.$ ) absorption bands in IR spectra were observed. Compound 1 was identified as ajugol when comparing ${ }^{1} \mathrm{H}$ and ${ }^{13} \mathrm{C}$ NMR spectra with those of ajugol. ${ }^{19}$

Compound 2 (see Figure 1) was isolated as white amorphous powder with the molecular formula $\mathrm{C}_{15} \mathrm{H}_{22} \mathrm{O}_{9}$ (LC-ESIMS $\mathrm{m} / \mathrm{z}$ $\left.369[\mathrm{M}+\mathrm{Na}]^{+}\right)$. An iridoid enolether system (205 $\left.\mathrm{nm}\right)$ in UV spectrum; hydroxyl group (3275 $\mathrm{cm}^{-1}$ ) and double-bond (1650 $\mathrm{cm}^{-1}$ ) absorption bands in IR spectra were observed. Compound 2 was identified as aucubin when comparing ${ }^{1} \mathrm{H}$ and ${ }^{13} \mathrm{C}$ NMR spectra with those of aucubin. ${ }^{20,21}$

Compound 3 (see Figure 1) was isolated as a white amorphous powder with the molecular formula $\mathrm{C}_{30} \mathrm{H}_{38} \mathrm{O}_{15}$ (LC-ESIMS $\mathrm{m} / \mathrm{z}$ $661[\mathrm{M}+\mathrm{Na}]^{+}$). The presence of an iridoid enolether system (216 $\mathrm{nm}$ ) and an aromatic acid (277 nm) moiety in UV spectrum and absorption bands for a hydroxyl group (3405 $\left.\mathrm{cm}^{-1}\right)$, a conjugated ester carbonyl (1704 $\left.\mathrm{cm}^{-1}\right)$, a double-bond $\left(1655 \mathrm{~cm}^{-1}\right)$ and an aromatic ring $\left(1451 \mathrm{~cm}^{-1}, 1508 \mathrm{~cm}^{-1}\right)$ in IR spectra were observed. The ${ }^{1} \mathrm{H}$ and ${ }^{13} \mathrm{C}$ NMR spectra of 3 were similar to those of lasianthoside I. Based on this evidence, compound 3 was identified as lasianthoside I. $^{22}$

Compound 4 (Figure 1) was isolated as a white amorphous powder with the molecular formula $\mathrm{C}_{15} \mathrm{H}_{22} \mathrm{O}_{10}$ (LC-ESIMS $\mathrm{m} / \mathrm{z}$ $\left.385[\mathrm{M}+\mathrm{Na}]^{+}\right)$. Its UV spectrum supported the presence of an iridoid enolether system (208 $\mathrm{nm}$ ) and absorption bands were for a hydroxyl group $\left(3450 \mathrm{~cm}^{-1}\right)$, and a double-bond $\left(1670 \mathrm{~cm}^{-1}\right)$

Table 4. Minimum inhibitory concentrations $(\mu \mathrm{g} / \mathrm{mL})$ of the methanolic extract and the secondary metabolites

\begin{tabular}{|c|c|c|c|c|c|c|c|}
\hline & \multicolumn{4}{|l|}{ Bacteria } & \multicolumn{3}{|l|}{ Fungi } \\
\hline & $\begin{array}{l}\text { Staphylococcus } \\
\text { aureus }\end{array}$ & $\begin{array}{l}\text { Enterococcus } \\
\text { faecalis }\end{array}$ & $\begin{array}{l}\text { Escherichia } \\
\text { coli }\end{array}$ & $\begin{array}{l}\text { Pseudomonas } \\
\text { aeruginosa }\end{array}$ & $\begin{array}{l}\text { Candida } \\
\text { albicans }\end{array}$ & $\begin{array}{l}\text { Candida } \\
\text { krusei }\end{array}$ & $\begin{array}{l}\text { Candida } \\
\text { parapsilosis }\end{array}$ \\
\hline & ATCC 29213 & ATCC 29212 & ATCC 25922 & ATCC 27853 & ATCC 90028 & ATCC 6258 & ATCC 22019 \\
\hline $\begin{array}{l}\text { Verbascum mucronatum-MeOH } \\
\text { extract }\end{array}$ & 256 & 128 & 256 & 256 & 256 & 128 & 128 \\
\hline Ajugol & 128 & 256 & 128 & 128 & 64 & 128 & 64 \\
\hline Aucubin & 256 & 512 & 512 & 256 & 128 & 256 & 256 \\
\hline Lasianthoside I & $>512$ & 512 & 512 & 512 & 256 & 512 & 256 \\
\hline Catalpol & 256 & 512 & 512 & 256 & 256 & 256 & 256 \\
\hline Ilwensisaponin C & $>512$ & $>512$ & 512 & 512 & 256 & 512 & 256 \\
\hline Ilwensisaponin A & 256 & $>512$ & $>512$ & 512 & 64 & 64 & 128 \\
\hline Verbascoside & 256 & 512 & 512 & 256 & 256 & 256 & 256 \\
\hline Ampicillin & 1 & 8 & 2 & - & - & - & - \\
\hline Fluconazole & - & - & - & - & 1 & 64 & 8 \\
\hline
\end{tabular}


in the IR spectra were observed. The ${ }^{1} \mathrm{H}$ and ${ }^{13} \mathrm{C}$ NMR spectra of compound $\mathbf{4}$ were similar to those of catalpol. Thus, compound 4 was identified as catalpol. ${ }^{23}$

Compounds 5 and 6 (Figure 1) were obtained as amorphous compounds with molecular weights of 1104 \{LC-ESIMS: $\mathrm{m} / z$ $\left.1127\left([\mathrm{M}+\mathrm{Na}]^{+}\right)\right\}$, and $1072\left\{\right.$ LC-ESIMS: $\left.\mathrm{m} / z 1095\left([\mathrm{M}+\mathrm{Na}]^{+}\right)\right\}$, as calculated for $\mathrm{C}_{55} \mathrm{H}_{92} \mathrm{O}_{22}$ and $\mathrm{C}_{54} \mathrm{H}_{88} \mathrm{O}_{21}$, respectively.

In their IR spectra, the observed absorbances were consistent with the presence of olefinic double bonds. The ${ }^{1} \mathrm{H}$ and ${ }^{13} \mathrm{C}$ NMR data of compounds 5 and 6 suggested that they had similar structures, possessing the same sugar moieties but differing in their aglycones.

In the ${ }^{1} \mathrm{H}$ NMR spectrum of compound 5 , characteristic resonances for anomeric protons were observed at $\delta_{H} 4.91(d$, $J=6.6 \mathrm{~Hz}), 5.21(d, J=7.0 \mathrm{~Hz}), 5.54(d, J=7.0 \mathrm{~Hz}), 5.78(\mathrm{bs})$, and, in the ${ }^{13} \mathrm{C}$ NMR spectrum, anomeric carbons at $\delta_{c} 104.2(\beta-D-$ fucopyranose), 105.1 ( $\beta$-D-glucopyranose-inner), 104.0 ( $\beta$-Dglucopyranose-terminal) and 102.8 ( $\alpha$-L-rhamnopyranose), as well as 2 proton signals at $\delta_{H} 1.35(d, J=4.8 \mathrm{~Hz})$ and $1.68(d, J=$ $5.5 \mathrm{~Hz}$ ), arising from the secondary methyl groups in the sugar moieties. By means of HMBC correlations, the sequence of the saccharidic chain was determined as [ $\alpha$-L-rhamnopyranosyl$(1 \rightarrow 4)$ - $\beta$-D-glucopyranosyl- $(1 \rightarrow 3)]$-[ $\beta$-D-glucopyranosyl$(1 \rightarrow 2)]-\beta$-D fucopyranoside.

The ${ }^{1} \mathrm{H}$ NMR of compound $\mathbf{5}$ showed 6 tertiary methyl signals at $\delta_{H} 0.88,0.95,0.96,1.07,1.08$ and 1.30 . The proton signal at $\delta_{H}$ $3.21(3 \mathrm{H})$ was attributed to methoxy protons, and $\delta_{H} 5.46(\mathrm{br} \mathrm{s})$ to the olefinic proton of the aglycone. It was determined that the aglycone was an oleanane- $\Delta^{12}$ type confirmed by presence of $\delta_{c}$ 122.6 and 148.1 signals in the ${ }^{13} \mathrm{C}$ NMR spectrum. The assignment of the remaining NMR signals was achieved by means of ${ }^{1} \mathrm{H}-{ }^{1} \mathrm{H}$ COSY, HMQC, and HMBC experiments.

The location of the methoxy group was determined using HMBC correlations between methoxy protons and $\mathrm{C}-11$, whereas a chemical shift of $\mathrm{C}-11\left(\delta_{c}\right.$ 76.2) was also evident. From the chemical shift of $\mathrm{C}-11\left(\delta_{c} 76.2\right)$ in compound 5 , it can be concluded that the methoxyl group had an $\alpha$-configuration as reported for saikosaponin- $\mathrm{b}_{4}{ }^{24}$ The $\mathrm{H}-3$ methine proton, $\mathrm{H}-23$ and $\mathrm{H}-28$ methylene protons showed downfield shifts due to hydroxy substitutions.

Consequently, the structure was elucidated to be $3-0-\{[\alpha-$ L-rhamnosyl-( $1 \rightarrow 4)$ - ( $\beta$-D-glucopyranosyl- $(1 \rightarrow 3)]-\beta$-Dglucopyranosyl]-(1 $\rightarrow 2)$ - $\beta$-D-fucopyranosyl-11-methoxy-olean12-ene-3 $3,23,28$-triol (=ilwensisaponin C). ${ }^{25}$

Compound 6 was distinguished from compound 5 by differences in the aglycone parts in ${ }^{1} \mathrm{H}$ and ${ }^{13} \mathrm{C}$ NMR spectra.

The ${ }^{1} \mathrm{H}$ NMR of compound 6 showed 6 tertiary methyl signals at $\delta_{H} 0.82,0.87,0.96,0.98,1.04$ and 1.31 . The olefinic protons $\mathrm{H}-11$ and $\mathrm{H}-12$ were determined at $5.94(\mathrm{br} \mathrm{d}, \mathrm{J}=10.4 \mathrm{~Hz}), \delta_{c} 132.9$ and $\delta_{H} 5.53\left(^{*}\right), \delta_{c} 131.9$, respectively. Thus, aglycone was identified as an oleanane- $\Delta^{11}$ type and no signals of a methoxy group in ${ }^{1} \mathrm{H}$ and ${ }^{13} \mathrm{C}$ NMR spectra of compound 6 were observed compared with those of compound 5 .
Due to presence of an oxo-bridge between C-28 and C-13, a chemical shift of $\mathrm{C}-28$ methylene protons $\left(\delta_{H} 3.33-3.72\right)$ appeared in the higher field in comparison with those of $\mathrm{C}-23$ hydroxylated methylene protons $\left(\delta_{H} 3.70-4.34\right)$. Based on this evidence, the aglycone of compound 6 was determined as $13 \beta$, 28-epoxyolean-11-ene-3 $\beta, 23$-diol. ${ }^{26}$

As a result, the structure of compound 6 was determined as

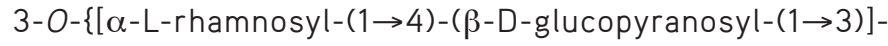
$\beta$-D-glucopyranosyl]-(1 $\rightarrow 2)-\beta$-D-fucopyranosyl\}-13 $\beta, 28$ epoxyolean-11-ene-3 $\beta, 23$-diol (=ilwensisaponin $A^{25}=$ mimengoside $A)^{27}$

Compound 7 (Figure 1) was obtained as an amorphous powder. Its structure was identified as verbascoside by comparing its ${ }^{1} \mathrm{H}$ and DEPT- ${ }^{13} \mathrm{C}$ NMR data with previously published data and by direct comparison with the authentic sample on a TLC plate.

It has been reported that Verbascum L. species contained diverse iridoid glycosides such as ajugol ${ }^{5,13}$, aucubin ${ }^{28}$, lasianthoside ${ }^{22}$ and catalpol ${ }^{23}$; saponins such as ilwensisaponin $\mathrm{C}^{13}$ and ilwensisaponin $A^{13}$; and phenylethanoid glycosides such as verbascoside. ${ }^{13}$ Ilwensisaponin A has previously been found to be active against Aspergillus fumigatus; ${ }^{29}$ it showed moderate antifungal activity in the current study.

\section{CONCLUSIONS}

This paper is the first to report the presence of these compounds from $V$. mucronatum Lam. Our continuing studies will be of assistance in clarifying the chemotaxonomic classification of the genus Verbascum L. On the other hand, when the antimicrobial activity results were evaluated, the higher activities of ajugol and ilwensisaponin $A$ than the $V$. mucronatum extract suggest that more active compounds may be found in further phytochemical studies.

\section{ACKNOWLEDGEMENTS}

The authors would like to thank Prof. Dr. Hayri Duman, Gazi University, Faculty of Science, Department of Botany, Etiler, Ankara, Turkey, for the authentication of the plant specimen.

Conflict of Interest: No conflict of interest was declared by the authors.

\section{REFERENCES}

1. Tutin TG. Flora Europaea Vol 3. Cambridge; University Press; 1972.

2. Davis PH, Mill RR, Tan K. Flora of Turkey and the East Aegean Islands. Edinburg; University Press; 1988.

3. Ekim T, Verbascum L. In: Güner A, Özhatay N, Ekim T, Başer KHC, eds. Flora of Turkey and East Aegean Islands. Edinburg University Press; 2000:193-194.

4. Huber-Morath A, Verbascum L. In: Davis P, ed. Flora of Turkey and the East Aegean Islands. Edinburgh University Press; 1978:461-463.

5. Baytop A. Therapy with Medicinal Plants in Turkey (Past and Present). Nobel Tip Kitabevleri Ltd; 1999.

6. Sezik E, Yeșilada E, Honda G, Takaishi Y, Takeda Y, Tanaka T. Traditional medicine in Turkey X. Folk medicine in Central Anatolia. J Ethnopharmacol. 2001;75:95-115. 
7. Tuzlaci E, Alparslan DF. Turkish folk medicinal plants, part V: Babaeski (Kırklareli). J Pharm Istanbul University. 2007;39:11-23.

8. Turker AU, Camper ND. Biological activity of common mullein, a medicinal plant. J Ethnopharmacol. 2002;82:117-125.

9. Tatli I, Akdemir ZS. Traditional uses and biological activities of Verbascum species. FABAD J Pharm Sci. 2006;31:85-96.

10. Kahraman C, Ekizoglu M, Kart D, Akdemir ZS, Tatli I. Antimicrobial activity of some Verbascum species growing in Turkey. FABAD J Pharm Sci. 2011;36:11-15.

11. Cubukcu B, Atay M, Sarıyar G, Ozhatay N. Folk medicines in Aydın. Journal of Traditional and Folcloric Drugs. 1994;1:1-58.

12. Akdemir ZS, Tatli I, Bedir E, Khan IA. Two new iridoid glucosides from Verbascum salviifolium Boiss. Z Naturforsch B. 2005;60:113-117.

13. Tatli I, Akdemir ZŞ, Bedir E, Khan IA. Saponin, iridoid, phenylethanoid and monoterpene glycosides from Verbascum pterocalycinum var. mutense. Turk J Chem. 2004;28:111-122.

14. Akdemir ZŞ, Tatli I, Bedir E, Khan IA. Neolignan and phenylethanoid glycosides from Verbascum salviifolium boiss. Turk J Chem. 2004;28:621628.

15. Akdemir ZS, Tatli I, Bedir E, Khan IA. Iridoid and phenylethanoid glycosides from Verbascum lasianthum. Turk J Chem. 2004:28:227-234.

16. Akdemir ZS, Tatli II, Bedir E, Khan IA. Antioxidant flavonoids from Verbascum salviifolium boiss. FABAD J Pharm Sci. 2004;28:71-75.

17. Wayne P. Reference method for broth dilution antifungal susceptibility testing of yeasts: Approved standard. 3rd ed. M 27-A3 ed: Clinical and Laboratory Standards Institute; 2008.

18. Wayne P. Methods for dilution antimicrobial susceptibility tests for bacteria that grow aerobically: Approved standard. 8th ed. M 07-A8 ed: Clinical and Laboratory Standards Institute; 2008.
19. Pardo F, Perich F, Torres R, Delle Monache F. Phytotoxic iridoid glucosides from the roots of Verbascum thapsus. J Chem Ecol. 1998;24:645-653.

20. Bianco A, Passacantilli P, Polidori G. $1 \mathrm{H}$ and 13CNMR data of C-6 epimeric iridoids. Org Magn Resonance. 1983;21:460-461.

21. Chaudhuri RK, Sticher O. New iridoid glucosides and a lignan diglucoside from Globularia alypum L. Helv Chim Acta. 1981;64:3-15.

22. Tatli I, Khan IA, Akdemir ZS. Acylated iridoid glycosides from the flowers of Verbascum lasianthum Boiss. ex Bentham. Z Naturforsch B. 2006;61:1183-1187.

23. Tatlil,Akdemir ZS,Bedir E,KhanIA.6-O-alpha-L-rhamnopyranosylcatalpol derivative iridoids from Verbascum cilicicum. Turk J Chem. 2003;27:765772 .

24. Ishii H, Seo S, Tori K, Tozyo T, Yoshimura Y. The Structures of Saikosaponin-E and Acetylsaikosaponins, minor components isolated from Bupleurum falcatum L. determined by C-13 Nmr-spectroscopy. Tetrahedron Lett. 1977:1227-1230.

25. Calis I, Zor M, Basaran AA, Wright AD, Sticher O. Ilwensisaponin A, B, $C$ and triterpene saponins from Scrophularia ilwensis. Helv Chim Acta. 1993;76:1352-1360.

26. Tori K, Yoshimura Y, Seo S, Sakurawi K, Tomita Y, Ishii H. Carbon-13 NMR spektra of saikogenins. Stereochemical dependence in hydroxilation effects upon carbon-13 chemical shifts of oleanene-type triterpenoids. Tetrahedron Lett. 1976;17:4163-4166.

27. Ding N, Yahara S, Nohara T. Structure of mimengosides A and B, new triterpenoid glycosides from Buddleja flos produced in China. Chem Pharm Bull. 1992;40:780-782.

28. Akdemir ZS, Tatli I, Bedir E, Khan IA. Acylated iridoid glycosides from Verbascum lasianthum. Turk J Chem. 2004;28:101-109.

29. Tatlı I, Akdemir Z. Antimicrobial and antimalarial activities of secondary metabolites from some Turkish Verbascum species. FABAD J Pharm Sci. 2005;30:84-92. 